\title{
CDISC CDASH Unit for Duration of Treatment Interruption Terminology
}

National Cancer Institute

\section{Source}

National Cancer Institute. CDISC CDASH Unit for Duration of Treatment Interruption

Terminology. NCI Thesaurus. Code C78427.

A terminology subset of the CDISC Study Data Tabulation Model (SDT M) Unit codelist created for Clinical Data Acquisition Standards Harmonization (CDASH) Unit for Duration of Treatment Interruption terminology. 